(C) [2004] IEEE. Reprinted, with permission, from [Ananda S. Mohan, A Circularly Polarised Stacked Electromagnetically Coupled Patch Antenna, Antennas and Propagation, IEEE Transactions on (Volume:52 , Issue: 5 ), May 2004]. This material is posted here with permission of the IEEE. Such permission of the IEEE does not in any way imply IEEE endorsement of any of the University of Technology, Sydney's products or services. Internal or personal use of this material is permitted. However, permission to reprint/republish this material for advertising or promotional purposes or for creating new collective works for resale or redistribution must be obtained from the IEEE by writing to pubs-permissions@ieee.org. By choosing to view this document, you agree to all provisions of the copyright laws protecting it 


\section{A Circularly Polarised Stacked Electromagnetically Coupled Patch Antenna}

Kwok L. Chung and Ananda S. Mohan

\begin{abstract}
In this paper, we present a high-performance circularly polarised (CP) stacked electromagnetically coupled patch antenna and its subarray at $\mathrm{X}$-band. In addition to low boresight axial-ratios, the subarray has measured 10-dB impedance and 3dB axial-ratio bandwidths of $25.6 \%$ and $23.5 \%$ respectively as compared to the measured $20.2 \%$ and $8.0 \%$ for a single element. The mutual coupling for this element is shown to be lower than other reported stacked patch antennas and obtained a gain (>10dbic) bandwidth of $23.5 \%$. The calculated antenna efficiency is $89 \%$ around centre frequency for the single element whereas the subarray has an overall efficiency of $>71 \%(-1.5 \mathrm{~dB})$ over $21 \%$ bandwidth.
\end{abstract}

Index Terms-Antenna efficiency; axial-ratio bandwidth $\left(A_{x} B W\right)$; EMCP antennas; singly-fed circularly polarised (SFCP) patch antennas; stacked patch antennas.

\section{INTRODUCTION}

Traditional single layer, singly-fed circularly polarised (SFCP) microstrip patch antennas have inherent limitations in gain, impedance and axial-ratio bandwidths. This is mainly owing to the resonant nature of the patch antennas - a high unloaded Q-factor and the frequency-dependent excitation of the two degenerative modes $\left(\mathrm{TM}_{01}\right.$ and $\left.\mathrm{TM}_{10}\right)$ when using a single feed. One way to increase the impedance bandwidth is by minimising the unloaded Q-factor with the use of an electrically thick substrate for the patch. However, the feed reactance and surface-wave power due to the thick substrate can become additional problems. As an alternative to increase the gain and bandwidth, the stacked patches and electromagnetically coupled patch (EMCP) configurations for linear polarisation (LP) were proposed [1]-[2]. The surface-wave problems can be overcome either by the use of $\mathrm{Lo}-\mathrm{Hi}$ (substratesuperstrate) dielectric layer combination [3] or having an electrically large printed patch particularly for complex structures [4]. However, these methods can result in complicated antenna structures with limited bandwidth improvement. Owing to the larger patch, to build an array composed of such elements having a centre spacing of less than $0.7 \lambda_{0}$ can be difficult. Waterhouse proposed a technique that uses stacked patches printed on $\mathrm{Hi}$ - $\mathrm{Lo}$ dielectric layers (two-layers) to obtain not only high surface-wave efficiency but also broad impedance bandwidth $(\mathrm{ZBW})$ and axial-ratio bandwidth $\left(\mathrm{A}_{\mathrm{x}} \mathrm{BW}\right)$ for the $\mathrm{CP}$ patch antennas [5]. The fundamental basis for the technique in [5] is that the patch antennas require loosely bound field for radiation into space, while circuitry and feed

Manuscript received March 18, 2003; revised June 14, 2003. This work was carried out with financial support from the Commonwealth of Australia through the Cooperative Research Centres Program.

The authors are with the Microwave and Wireless Technology Research Group, ICT Group, Faculty of Engineering, University of Technology, Sydney, NSW 2007, Australia. network require tightly bound fields to suppress undesired radiation and coupling. Therefore, the use of high dielectric constant for substrate and low dielectric constant for superstrate becomes a straightforward choice. Based on this and those available in [5], the authors have developed a design method to optimise the SFCP-EMCP antennas with sandwich $\mathrm{Hi}$-Lo-Lo structure to have either a broad 3-dB axial-ratio bandwidth $\left(\mathrm{A}_{\mathrm{x}} \mathrm{BW}\right)$ or a low boresight axial ratio [10]. A unique feature of the method shown in [10] is that the mean frequencies of the corresponding bandwidths can be tightly controlled to be close to the centre frequency, which helps to guarantee a broadband performance for the optimised antenna.

In this paper, we present a unique design of SFCP-EMCP antenna having a zero perturbation on the parasitic (top) patch and show its performance in a $2 \times 2$ array configuration at Xband. First, the single element is designed by using the design and tuning technique reported in [10]. Next using the wellknown sequential feeding technique [6], a 4-element planar array is designed. We have obtained in addition to a low axialratio of about $0.3 \mathrm{~dB}$ around the centre frequency, a gain bandwidth ( $>10 \mathrm{dBic})$ in excess of $23.5 \%$, a $10-\mathrm{dB}$ impedance bandwidth (ZBW) of $25.6 \%$ and a $3-\mathrm{dB}$ axial-ratio bandwidth $\left(\mathrm{A}_{\mathrm{x}} \mathrm{BW}\right)$ of $23.5 \%$ for the proposed array. The overall efficiency for both the single element and the subarray have been calculated to be as high as $89 \%(-0.5 \mathrm{~dB})$ at $10 \mathrm{GHz}$. The axialratios within the bandwidth are also verified by inspecting the cross-polar levels from the measured radiation patterns.

\section{ANTENNA GEOMETRY AND DESIGN STRATEGy}

The proposed element is a right-hand circularly polarised (RHCP) design with a desired centre frequency of $10 \mathrm{GHz}$. The laminate selected for the substrate here is Rogers ${ }^{\circledR}$ RO3006 $\left(\varepsilon_{\mathrm{r}}=6.15, \tan \delta=0.002\right)$ with a thickness of $0.64 \mathrm{~mm}$ $\left(0.021 \lambda_{\mathrm{o}}\right)$ whereas the superstrate is RT/duriod $5880\left(\varepsilon_{\mathrm{r}}=2.2\right.$, $\tan \delta=0.0009)$ with a thickness of $0.79 \mathrm{~mm}\left(0.026 \lambda_{\mathrm{o}}\right)$, the height of second layer, $d_{2}$, is optimised to $3.3 \mathrm{~mm}\left(0.11 \lambda_{\mathrm{o}}\right)$ in order to yield a low axial-ratio. The shapes of the stacked patches with all the dimensions are shown in Fig. 1, where $\lambda^{\mathrm{H}}$, $\lambda^{\mathrm{L}}$ are the microstrip patch wavelength in substrate and super-

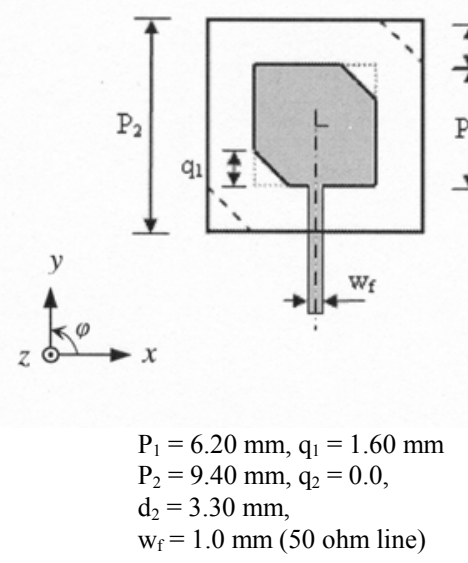

Fig. 1. X-band RHCP-EMCP element: geometry and dimensions of the stacked patches. 
strate respectively, and $\lambda_{\mathrm{o}}$ is free-space wavelength at the centre frequency. The parasitic patch in the proposed antenna element has zero perturbation (i.e. becomes a square patch) and is a modified version of that reported in [10].

We have used a material with a dielectric constant of 6.15 instead of 10.2 for the substrate as a compromise amongst CP performance, dielectric losses and the manufacturing tolerances over X-band. More importantly, the stability of the dielectric constant of RO3006 [8] over the frequency range is very critical to achieve a reliable design. Recently, a LP highperformance probe-fed stacked circular patch antenna at Lband was reported in [9]. The authors reported a surface-wave efficiency of greater than $80 \%$ and an impedance bandwidth of about $40 \%$ by using a material with a dielectric constant of around 5 for the grounded substrate whereas their second substrate has a dielectric constant close to the free-space. However, the copper and dielectric losses become critical at Xband than at lower frequencies. The antenna efficiency calculated for $\mathrm{CP}$ antennas presented here include all the loss mechanisms at X-band. All tuning and simulations were done using Ansoft Ensemble ${ }^{\mathrm{TM}}$. The measured and simulated results for the single element are plotted together in Figs. 3 and 4 for comparison. The measured minimum axial-ratio is $0.3 \mathrm{~dB}$ whereas the measured gain is slightly higher than the simulated one. The single element has a measured 10-dB ZBW of $20.2 \%$ and a $3-\mathrm{dB} \mathrm{A}_{\mathrm{x}} \mathrm{BW}$ of $8.0 \%$ with a mean frequency of $9.62 \mathrm{GHz}$. There is a shift of about $-3.3 \%$ in measured $\mathrm{A}_{\mathrm{x}} \mathrm{BW}$ which could be due to the fabrication tolerances. However, this frequency shift would compensate for the shift in mean frequencies due to the effects of mutual coupling [7].

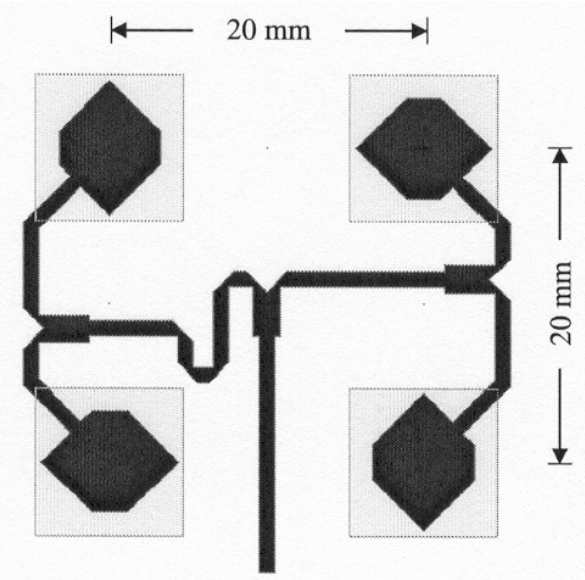

Fig. 2. Silhouette of $90^{\circ}$ sequentially rotated subarray composed of rotated elements with offset patches.

To design a $2 \times 2$ array (Fig. 2), we apply a sequential rotation of $90^{\circ}$ to 4 elements whose driven patches have $45^{\circ}$ offset. Such offset/rotation makes arraying into sequential rotation more efficient when the element spacing is small. Our results in Figs. 3 and 4 show that when the top-patch (P2) has a rotation of $45^{\circ}$ about z-axis, all the bandwidths and CP characteristics are almost unchanged. It has been reported that the smaller the element spacing the better the results in gain

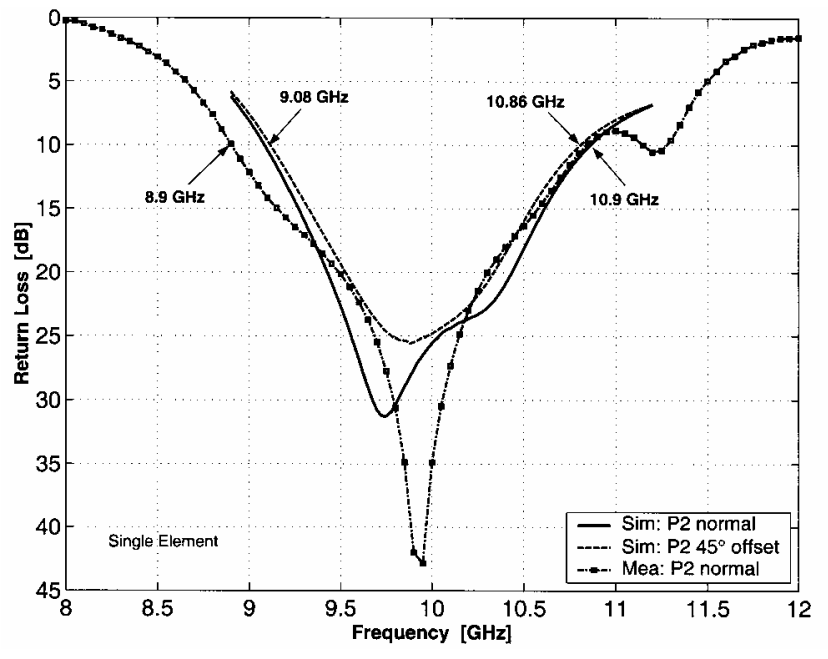

Fig. 3. Simulated and measured return loss of the single X-band RHCPEMCP element.

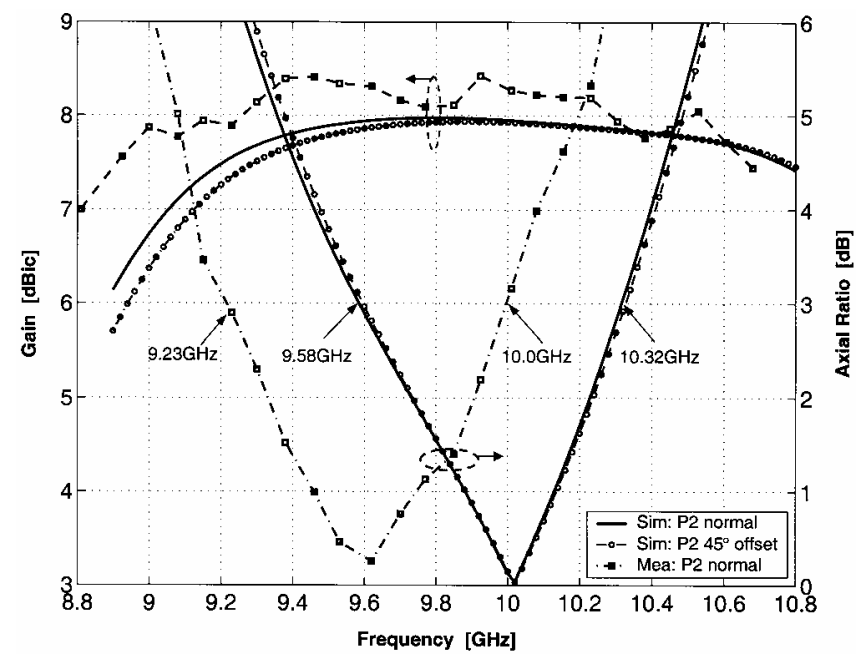

Fig. 4. Simulated and measured gain and axial-ratio of the single X-band RHCP-EMCP element.

bandwidth and suppression of grating lobes [6]. Our results to be shown in next section indicate that a smaller spacing also obtains a higher efficiency because of the shorter microstrip lines used to connect the driven patches. However, the small element spacing increases the mutual coupling and also makes it difficult to physically accommodate the $180^{\circ}$ phase-shifter within the available space. To locate the minimum element spacing in the array, we consider a simple two-element array that have the same geometry as shown in Fig. 1 but fed by a microstrip feedline of one-quarter guided wavelengths $\left(\lambda_{t}\right)$. We calculated the mutual coupling in both the planes using Ensemble $^{\mathrm{TM}}$, for a mutual coupling of $<-25 \mathrm{~dB}$, we obtain an element centre-spacing of $0.65 \lambda_{\mathrm{o}}$ and $0.6 \lambda_{\mathrm{o}}$ in collinear-plane (E-plane for LP antennas) and parallel-plane (H-plane for LP antennas), respectively. These values have been found to be better than those reported in literature for the EMCP and stacked patch configurations [2], [11]. After applying rotation to 4 elements with a square lattice of $20 \mathrm{~mm}\left(0.667 \lambda_{\mathrm{o}}\right)$, the 
mutual coupling between elements is expected to be lower than that of a normally oriented array since the combined Efield vectors for single elements become orthogonal to each other.

\section{EXPERIMENTAL RESULTS COMPARED TO SIMULATIONS}

All the measurements have been made inside an anechoic chamber; circular polarisation gain and axial ratio are measured by using a spinning horn and a gain comparison method. In spite of manufacturing tolerances, the measurements on the array, in general, are in good agreement with the simulated results as can be seen in Figs. 5 and 6. The measured minimum axial-ratio is $0.3 \mathrm{~dB}$ whereas the peak gain is about 12.5 $\mathrm{dBic}$ around the $10 \mathrm{GHz}$. The most significant result is the measured bandwidths are well overlaid with each other, which in turn leads to a useable bandwidth of about $21 \%$ with a mean frequency of $10.075 \mathrm{GHz}$ for this subarray. Table I given below summarises all the measured bandwidths for the $2 \times 2$ array.

It is well-known that patch antenna printed on a single-layer high dielectric constant material exhibits a low efficiency due to the excitation of surface-wave powers. The results from $\mathrm{Hi}$ Lo or Med-Lo structures in [5] and [9], however, showed a higher surface-wave efficiency of greater than $85 \%$ can be achieved. For the current design, which is a sandwich $(\mathrm{Hi}-\mathrm{Lo}-$ Lo) structure, we have used gain-directivity method to calculate the antenna efficiency using Ensemble ${ }^{\mathrm{TM}}$. The normalised radiation patterns for two principle and two diagonal cuts are

TABLE I

Summary of measured bandwidths for the $2 \times 2$ array

\begin{tabular}{c|c}
\hline $10 \mathrm{~dB} Z \mathrm{ZWW}$ & $8.60 \sim 11.12 \mathrm{GHz}$ \\
\hline $3 \mathrm{~dB} \mathrm{~A}_{\mathrm{x}} \mathrm{BW}$ & $8.80 \sim 11.14 \mathrm{GHz}$ \\
\hline $10 \mathrm{dBic} \mathrm{GBW}$ & $9.03 \sim 11.44 \mathrm{GHz}$ \\
\hline Overlaid BW & $9.03 \sim 11.12 \mathrm{GHz}$ \\
\hline
\end{tabular}

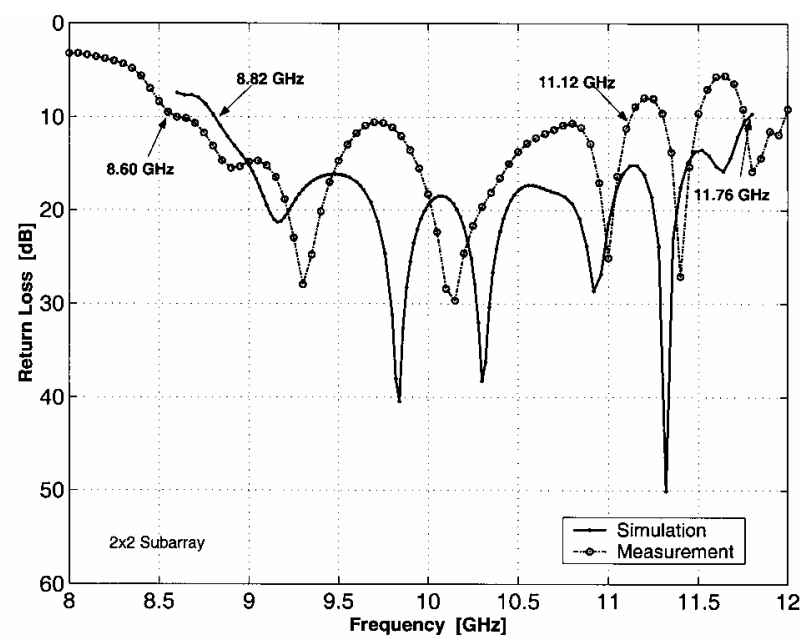

Fig. 5. Simulated and measured return loss of the $2 \times 2$ array. first computed for the selected frequency points and the directivity is then calculated by numerically integrating the field pattern data and dividing into $4 \pi$. The antenna efficiency in decibel is obtained by subtracting the directivity from the computed gain which is shown in Fig. 6. The antenna efficiency versus frequency has been calculated for three cases and plotted in Fig. 7. As expected the single element with $1 \lambda_{t}$ feedline has the highest efficiency in the band of 9.0 to 10.8 $\mathrm{GHz}$ while the efficiencies of $2 \times 2$ arrays with different feedline lengths $\left(1 \lambda_{t}\right.$ and $\left.5 \lambda_{t}\right)$ have shown significant differences. The antenna efficiency at $10 \mathrm{GHz}$ for the array with 5 $\lambda_{\mathrm{t}}$ feedline is $-1.04 \mathrm{~dB}(79 \%)$ compared to $-0.52 \mathrm{~dB}(89 \%)$ for the same array with $1 \lambda_{t}$ feedline, which is twice the value in $\mathrm{dB}$ of losses for an extra line-length of $4 \lambda_{\mathrm{t}}$ at the centre frequency. In the radiation pattern calculation, the size of finite ground-plane and dielectric materials, measurement and material tolerances are not taken into account, and hence the antenna efficiencies presented here can be regarded as the upperbound on the obtainable efficiency from the proposed antenna structure. As can be seen from Fig. 7, the array with $1 \lambda_{t}$

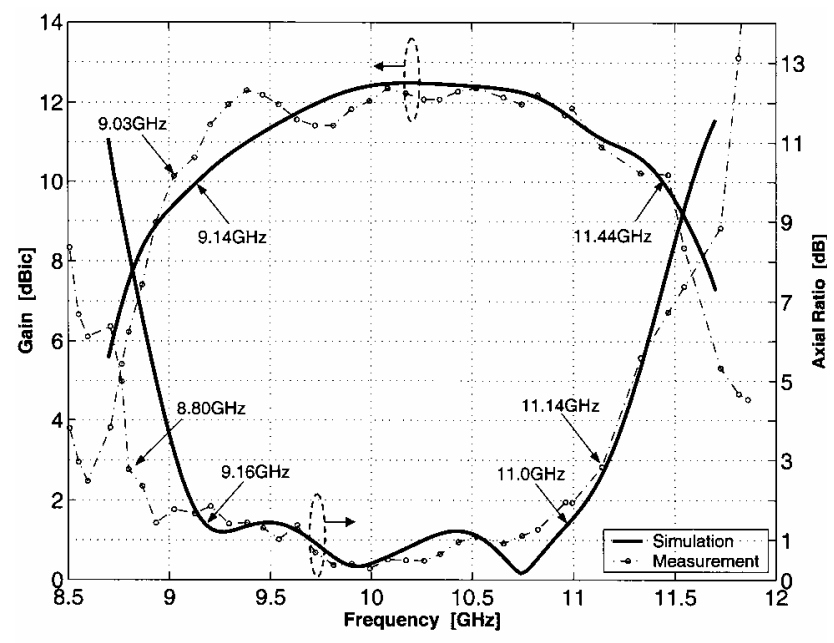

Fig. 6. Simulated and measured gain and axial-ratio of the $2 \times 2$ array.

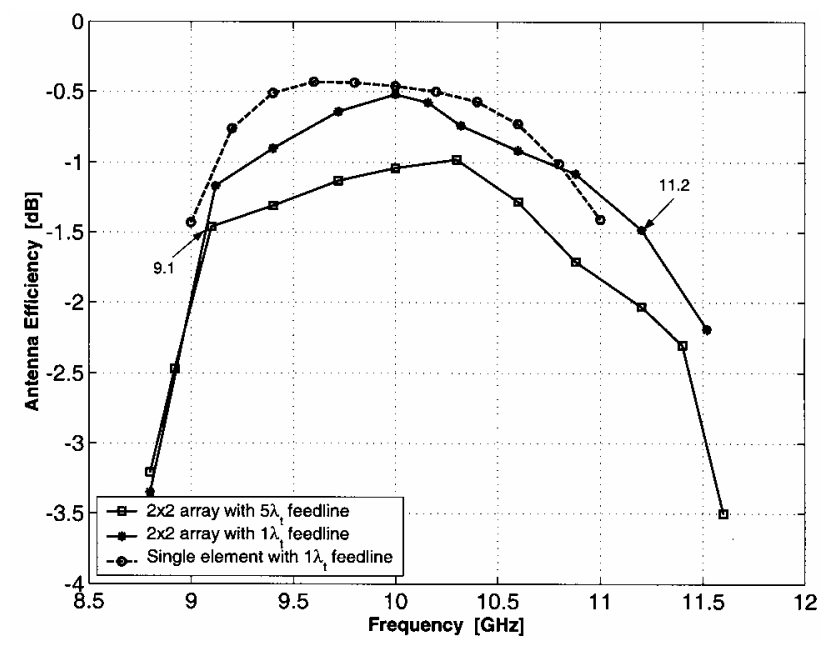

Fig. 7. Calculated antenna efficiency for single element and $2 \times 2$ array with different lengths of feedline. 
feedline has an efficiency of greater than $-1.5 \mathrm{~dB}(71 \%)$ over a bandwidth of 9.1 to $11.2 \mathrm{GHz}$. Fig. 8 shows the simulated and measured radiation pattern in $\varphi=0^{\circ}$ cut at $10 \mathrm{GHz}$. To verify the measured 3-dB axial-ratio bandwidth, the on-axis XPD of $>15 \mathrm{~dB}$ can be found from Figs. 9 and 10 in which both the simulated and measured patterns at 9 and $11 \mathrm{GHz}$, respectively, are plotted.

\section{CONCLUSION}

This paper presents a high-performance circularly polarised stacked patch antenna and a $2 \times 2$ subarray at X-band. We use high (6.15) and low (2.20) dielectric constant laminates for substrate and superstrate respectively in order to obtain a very low boresight axial-ratio $(0.3 \mathrm{~dB})$ for both a single element and a $2 \times 2$ array. The middle layer is formed by air dielectric

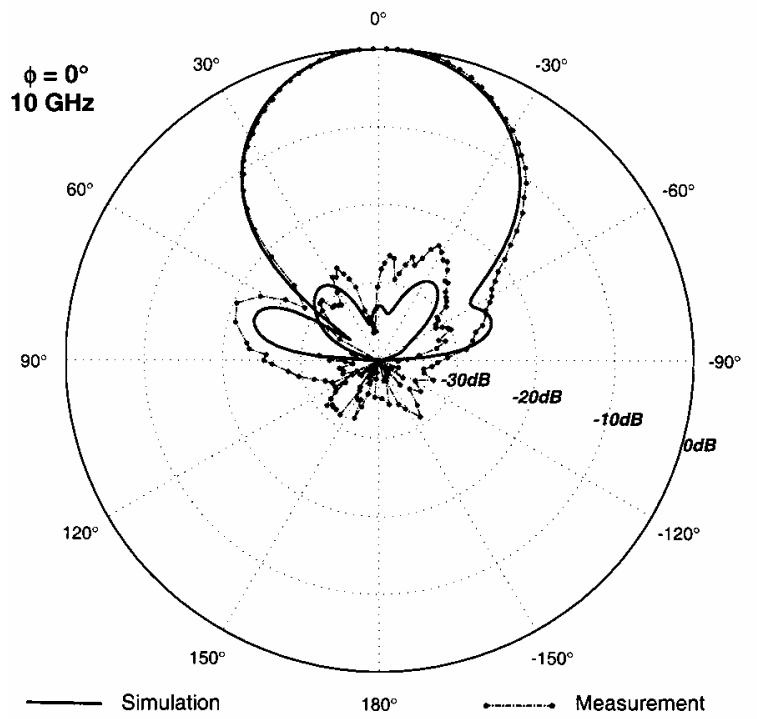

Fig. 8. Simulated and measured radiation pattern in $\varphi=0^{\circ}$ cut at $10 \mathrm{GHz}$.

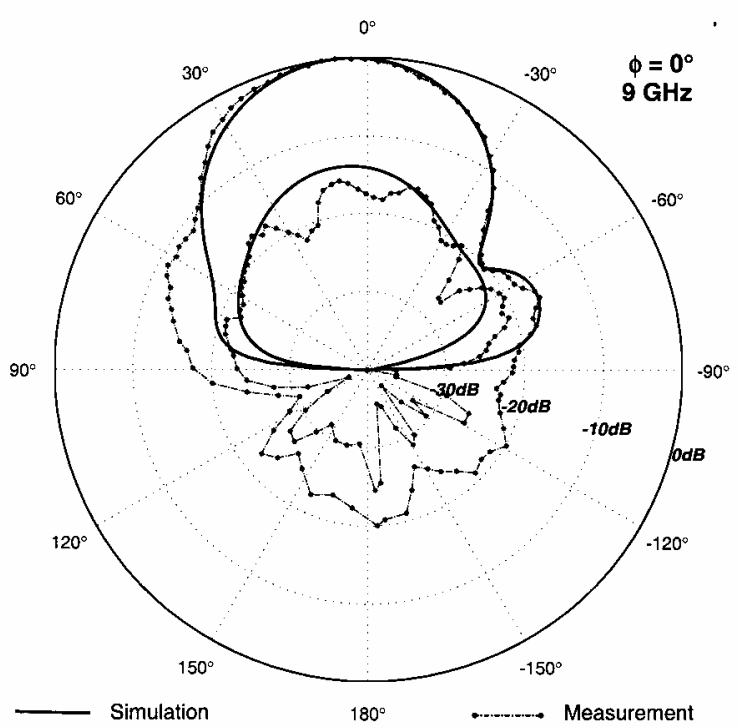

Fig. 9. Simulated and measured radiation pattern in $\varphi=0^{\circ}$ cut at $9 \mathrm{GHz}$. that effectively makes a stacked patch antenna having a $\mathrm{Hi}$ Lo-Lo structure for high performance. The array has a $10-\mathrm{dB}$ impedance and 3-dB axial-ratio measured bandwidths of $25.6 \%$ and $23.5 \%$ respectively as compared to the $20.2 \%$ and $8.0 \%$ for the single element. In addition to a circular polarisation gain of $12.5 \mathrm{dBic}$, a 10 -dBic gain bandwidth of $23.5 \%$ is obtained. The antenna efficiency is calculated to be greater than $-1.5 \mathrm{~dB}$ for a bandwidth of about $21 \%$ for both the single element and the subarray. The radiation patterns confirm the obtained axial-ratio bandwidth and the measured bandwidths are overlaid with each other.

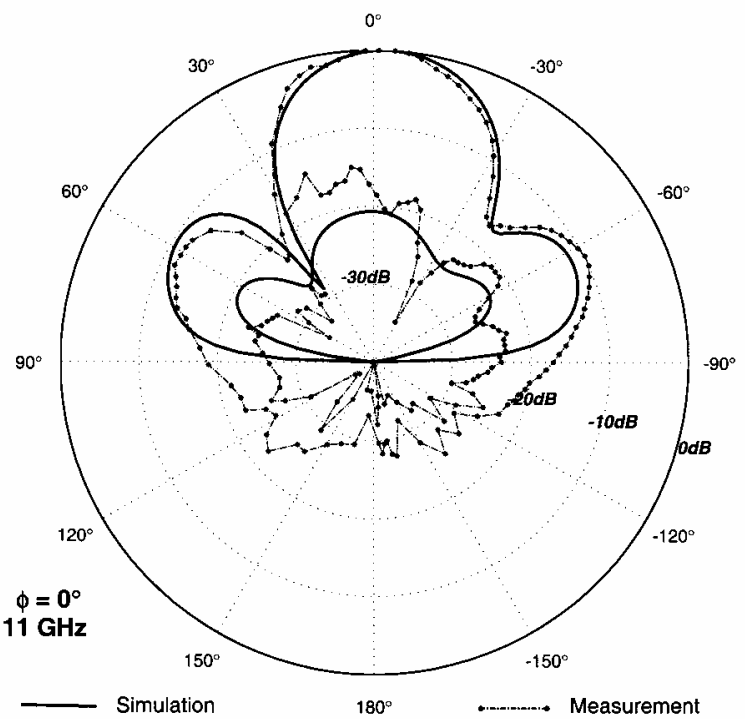

Fig. 10. Simulated and measured radiation pattern in $\varphi=0^{\circ}$ cut at $11 \mathrm{GHz}$.

\section{ACKNOWLEDGMENT}

We would like to thank Rogers ${ }^{\circledR}$ Corporation for the supply of dielectric material used in this study. We also thank the Antenna Group at CSIRO Telecommunications \& Industrial Physics for the use of their facilities and assistance for antenna measurements.

\section{REFERENCES}

[1] A. Sabban, "A new broadband stacked two-layer microstrip antenna", IEEE AP-S International Symposium Digest, 1983, pp. 63-66.

[2] R. Q. Lee and K. F. Lee, "Experimental study of the two-layer electromagnetically coupled rectangular patch antenna", IEEE Trans Antennas Propagat., vol. 38, pp. 1298-1302, Aug. 1990.

[3] N. G. Alexopoulos and D. R. Jackson, "Fundamental superstrate (cover) effects on printed circuit antennas", IEEE Trans Antennas Propagat., vol. AP-32, pp. 807-816, Aug. 1984.

[4] D. R. Jackson, J. T. Williams, A. K. Bhattacharyya, R. L. Smith, S. J. Buchheit and S. A. Long, "Microstrip patch designs that do not excite surface waves", IEEE Trans Antennas Propagat., vol. 41, pp. 10261037, Aug. 1993.

[5] R. B. Waterhouse, "Stacked patches using high and low dielectric constant material combinations", IEEE Trans Antennas Propagat., vol. 47, pp. 1767-1771, Dec. 1999.

[6] P. S. Hall, "Application of sequential feeding to wide bandwidth, circularly polarised microstrip patch arrays", in Inst. Elect. Eng. Proc. $H$, vol. 136, Oct. 1989 , pp. $390-398$. 
[7] U. R. Kraft, "An experimental study on $2 \times 2$ sequential-rotation arrays with circularly polarised microstrip radiators", IEEE Trans Antennas Propagat., vol. 45, pp. 1459-1466, Oct. 1997.

[8] Rogers ${ }^{\circledR}$ Technical Tip, "What is the maximum frequency at which I can use the various Rogers materials?", Available: http://www.rogers-corp.com/mwu/techindx.htm.

[9] A. Mitchell, M. Lech, D. M. Kokotoff and R. B. Waterhouse, "Search for high-performance probe-fed stacked patches using optimisation", IEEE Trans Antennas Propagat., vol. 51, pp. 249-255, Feb. 2003.

[10] K. L. Chung and A. S. Mohan, "A systematic design method to obtain broadband characteristics for singly-fed electromagnetically coupled patch antennas for circular polarisation", to appear in IEEE Trans. Antennas Propagat.

[11] C. Terret, S. Assailly, K. Mahdjoubi and M, Edimo, "Mutual coupling between stacked square microstrip antennas fed on their diagonal", Trans Antennas Propagat., vol. 39, pp. 1049-1051, July 1991. 